Case Reports

\title{
Using Lean Six Sigma to Increase Efficiency of a Grain Receipt Process of a Brazilian Agroindustry Cooperative
}

\author{
${ }^{1}$ Seiti Moraes Sakumoto, ${ }^{2}$ Daiane Maria De Genaro Chiroli and ${ }^{2}$ Ana Caroline Dzulinski \\ ${ }^{1}$ Department of Production Engineering, State University of Maringá, Maringá, Paraná, Brazil \\ ${ }^{2}$ Department of Production Engineering, Federal Technological University of Paraná, Ponta Grossa, Paraná, Brazil
}

\author{
Article history \\ Received: $14-12-2018$ \\ Revised: 05-01-2019 \\ Accepted: 14-06-2019 \\ Corresponding Author: \\ Daiane Maria De Genaro \\ Chiroli \\ Department of Production \\ Engineering, Federal \\ Technological University of \\ Paraná, Ponta Grossa, Paraná, \\ Brazil \\ Email: daianechiroli@utfpr.edu.br \\ dmgenaro@hotmail.com
}

\begin{abstract}
Due to the competitiveness of companies to increase market share, they have been more and more interested in topics related to cost reduction, process efficiency, new customers and continuous improvement. Considering this scenario, this study conducted analyses and implemented improvements using the Lean Six Sigma (LSS) methodology in a grain receipt process of a Brazilian agroindustrial cooperative to increase its profitability and consolidate a culture of continuous improvement in the cooperative. With the DMAIC steps, the Project Charter was used to describe the opportunities, to define the team and the macro schedule of the project; then SIPOC was used to understand the process, its inputs and outputs; the Requirements Tree was used to listen to the voice of the customer/business; Y Stratification was used to divide the work fronts; Process Mapping was used to identify the activities that do not add values; Cause and Effect Diagram and Matrix were used to identify and prioritize root causes; FMEA to analyze the risks and failure modes; and 5 Whys to generate an action plan, improvements were implemented in the bulk terminal that reduced the truck waiting time by 40 min for grain unloading; the quantity of truck inside the bulk terminal were also controlled with the upper limit of 60 trucks and the lower limit of 40 vehicles, so that the grain receipt process could improve its efficiency.
\end{abstract}

Keywords: Lean Six Sigma, Quality, Efficiency, Agroindustrial Cooperative

\section{Introduction}

Due to the current economic scenario, the companies are changing their management model to consolidate themselves in the market. Then, they are seeking innovation for their production processes aiming to reduce costs and eliminate waste (Snee, 2010), with quality as the fundamental aspect for achieving success (Laureani and Antony, 2016).

To ensure excellence in the quality of its products or services, the companies have to adapt themselves to the competitive environment and its requirements. Top management is investing strategically in technology and innovation, seeking to increase customer satisfaction (Dahlgaard and Dahlgaard-Park, 2006; Pisani et al., 2009).

Brazil has set new records in grain export in recent years; in 2016, the country estimated to export more than 100 million tons of soy and corn, corresponding to a $2 \%$ increase in relation to 2015 , developing the efficiency of its logistic operations and moving the
Brazilian economy to receive the products with minimized technical breakdown.

According to Barros (2006), investments in technology have increased the productivity of planted areas in agribusiness. The methodologies of Lean Manufacturing and Six Sigma optimize the receipt process to meet the new demand for products and are considered as highly effective tools (Tanik and Sen, 2012). Lean Manufacturing removes barriers to value flow in a process for faster processes, eliminates waste from critical processes, reduces the number of defective products/operations and the lead time for the delivery of the right product/service at the right time and at the right place (Antony, 2005a; 2005b). It focuses primarily on customer needs, process improvement and cost reduction (Domenech, 2016). It is a powerful business strategy that can improve quality in industries around the world (Kuvvetli and Firuzan, 2017).

The literature about lean six sigma has many important studies implemented in different countries and 
sectors (Kuvvetli and Firuzan, 2017; Sastry et al., 2011; Gupta and Bharti, 2013; Mamatha and Vasuki, 2014; Nakhai and Neves, 2009; Prashar, 2016; Tanik and Sen, 2012; Hilton et al., 2008; Zhang et al., 2012). Considering the relevance of this subject, this study conducted analyses and implemented improvements using the Lean Six Sigma methodology in a grain receipt process of a Brazilian agroindustrial cooperative to increase its profitability and consolidate a culture of continuous improvement in the cooperative.

This study has five sections. The first section presents the study context and purpose. The second describes the theoretical reference. The third and fourth describe the study methodology and results and discussion, respectively. The fifth section shows the final considerations.

\section{Literature Review}

The Lean Six Sigma methodology refers to two terms related to waste reduction and process control for continuous improvement of quality. Alhuraish et al. (2017), in their study about the success factors of the methodologies applied individually, report that regardless of the terms being analyzed together, both techniques aim to improve the processes favoring quality improvement of services or products. The authors also emphasize the techniques are relevant when the objective is the continuous improvement of processes. There is a fairly large and growing evidences associated with the benefits of implementing LSS (Fursule et al., 2012; Antony et al., 2016; Gijo et al., 2018). LSS program is a strategy that can lead to substantial gains in profitability and quality (Harry, 1998; Carvalho, 2002; Connor, 2003; Watson, 2003; Roberts, 2004; Snee, 2004).

Tenera and Pinto (2014) use Lean Six Sigma to propose a model for project management and emphasize the LSS methodology considers the DMAIC cycle (Define, Measure, Analyze, Improve and Control) together with the purpose of reducing defects and variations in production processes, leading to reduction of possible waste.

Alhuraish et al. (2017) identified 13 critical success factors in LSS: (1) Commitment and support from the top management, (2) education and training, (3) communication, (4) employee engagement, (5) culture change, (6) understanding of tools and techniques of lean manufacturing, (7) skills, (8) connection of the lean method with customers, (9) connection of the lean method with business strategy, (10) connection of the lean method with suppliers, (11) connection of the lean method with human resources, (12) rewards system and (13) project management skills. In other research has the list the following ones for the industry: Increase in benefits and savings, reduction in process defect levels, reduction of depot maintenance inspection time, reduction of turnaround time at repair shops, reduction of cycle time, increase in quality and productivity (Kwak and Anbari, 2006).

The theoretical analysis conducted in this research shows that all studies that have applied or evaluated LSS at some point highlight one of the factors mentioned by Alhuraish et al. (2017). Azadeh et al. (2016), for example, evaluates and optimizes the effect of Six Sigma implementation on working conditions of the employees of an automotive company, emphasizing aspects such as stress at work, satisfaction and safety at work, personal development and cooperation among employees.

Uluskan and Erginel (2017) study the Six Sigma life cycle to provide clues about the future of this methodology from a stochastic point of view by analyzing four dimensions: Cost, performance, enthusiasm and expectations. The autocorrelation function of Six Sigma processes converges to a function that shows a high level of correlation in the coming years. Finally, the authors give some suggestions so that the stationary stage achieved by Six Sigma can be always higher than the previous level and companies may inhibit the decline of Six Sigma.

Voelkel (2017), in a case study, analyzes the relationships between the process control tools and reliability of a manufactured product. For this purpose, tolerance intervals were set for statistical control based on optimization criteria. The result became very useful for both the supplier and the customer.

Pamfilie et al. (2012) emphasize the importance of leadership aspects when performing LSS actions. According to the authors, leadership drives other critical success factors such as development of the human resources, culture, education and training of the Organization.

One of the key elements that distinguish Lean Six Sigma from previous quality initiatives is the organization and structure of quality implementation functions (Laureani and Antony, 2016). Lean Six Sigma introduces a formal organizational infrastructure for different roles in quality implementation, lending terminology from the martial arts universe to define hierarchy and strategic paths to improve products and services (Adams et al., 2003; Antony et al., 2005a; 2005b; Harry and Schroeder, 2000; Pande et al., 2000; Snee, 2004).

Therefore, most studies that analyze LSS are focused on the effectiveness of actions and the contributions from the DMAIC applied for waste reduction, with actions influenced by the critical factors already mentioned.

This study is based on a case study of an activity of great economic impact in Brazil - agroindustrial production - and for this reason, studies with similar objectives were considered. Biazetto and Chiroli (2015), for instance, used the Six Sigma methodology in a grain warehouse due to increased commercial 
competitiveness and the need to improve current results, in order to identify and eliminate production losses. After data collection, the project team, through DMAIC steps, managed to minimize the main losses in the process from the solutions proposed by the project, reducing the waste during soybean transfer from a bulk terminal to an oil extraction plant.

To elucidate the steps of this case study, the next topic will detail the methodology selected according to the main objective.

\section{Methodology}

This study was developed in a grain receipt process in a bulk terminal of a Brazilian agroindustrial cooperative. Considering the large amount to be received and the importance of ensuring efficiency of this production process, it used Lean Six Sigma methodology with the help of the DMAIC steps, as shown in Fig. 1.

During the project, several tools were used, included in the DMAIC steps (Table 1). In the beginning, the Project Charter was used to describe the opportunities, to define the team and the macro schedule of the project; then SIPOC was used to understand the process, its inputs and outputs; the Requirements Tree was used to listen to the voice of the customer/business; Y Stratification was used to divide the work fronts; Process Mapping was used to identify the activities that do not add values; Cause and Effect Diagram and Matrix were used to identify and prioritize root causes; FMEA to analyze the risks and failure modes; and 5 Whys to generate an action plan.

\section{Development}

\section{Company Characterization}

This study was conducted in an agroindustrial cooperative in the south region of Brazil. Founded in 1963, it initially had 46 coffee growers and in its 56 years of history, it expanded now to 66 operating units, located in strategic points in the South, Central-west and Southeast regions of the country.

\section{Grain Receipt}

The cooperative has two types of grain receipts: The agricultural producer located nearby delivers the product directly to the cooperative, as it has exclusivity in a complex for members and the bulk terminal, which receives products from other operating units, called transfer stations, because all products received by producers nearby are later transferred to the unit analyzed in this study. The classification area is the initial stage of the grain receipt process; it classifies and establishes the waste of each cargo according to the results of the moisture and impurity analysis. In this area, four classifiers receive on average around 125 trucks every day in the harvest period. Therefore, a process without barriers in the flow of information has become increasingly necessary, justifying the development of this project.

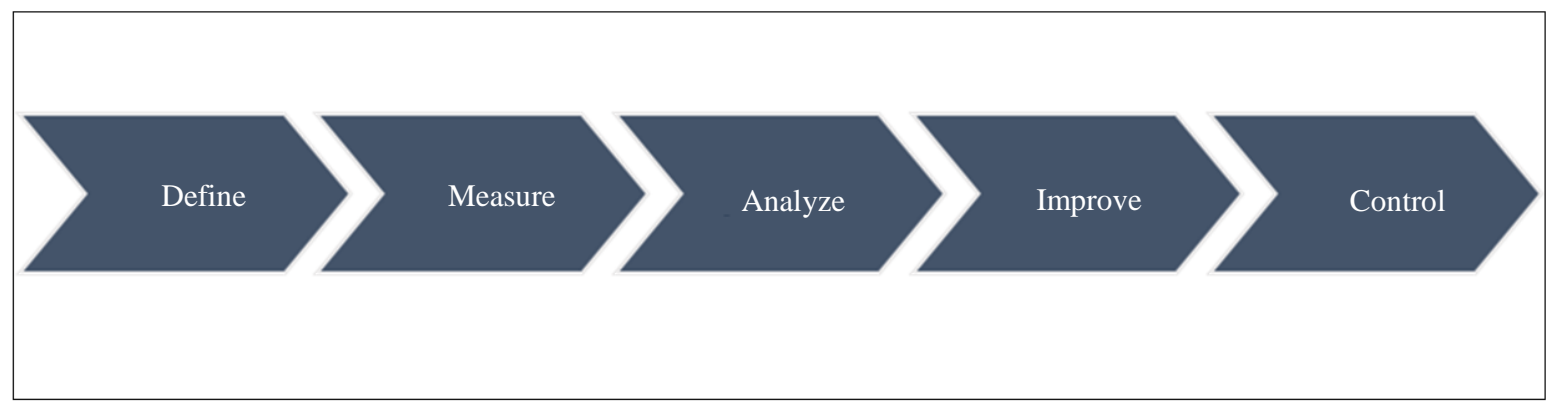

Fig. 1: Phases DMAIC; Define: Develop the scope of the team's work, define the macro map of the process, select customer requirements and analyze resistances; Measure: Map the process, analyze opportunities for process simplification, plan data collection, validate the measurement system and establish process capacity; Analyze: Analyze the chances of process redesign and identify the root causes that affect its performance; Improve: Advanced analysis of causes, find solutions, pilot and plan implementations of desired solutions; Control: Design new controls, conclude implementations and calculate their ability to analyze whether the result was achieved

Table 1: Time VA and NVA of the steps of receiving grains

\begin{tabular}{lcc}
\hline Phases & Time Value-Added (VA) (Minutes) & Time NVA (Value Not Added) (Minutes) \\
\hline Classification-balance entrance & 2 & 88 \\
Balance Input-Balance output & 13 & 37 \\
Total & 15 & 125 \\
\hline
\end{tabular}




\section{Lean Six Sigma Project}

A Lean Six Sigma project was conducted to enable the bulk terminal to meet the expected demand. This study to increase grain receipt was designed by the top management and a project office, linked with the strategic planning of the cooperative, aiming to improve process efficiency, optimizing the flow between the steps of the grain receipt process.

Define The cycle of the DMAIC methodology started with the Define step, establishing the business case that is linked with the strategic planning, the members of the project team and their dedication (time), in percentage of weekly hours, the opportunities, the goal and the macro schedule of the project phases.

The multidisciplinary team has 5 members and 3 experts. The project set the goal of an upper limit of 240 $\min (4 \mathrm{~h})$ to unload soybeans and $480 \mathrm{~min}(8 \mathrm{~h})$ to unload corn. First, the team developed the Project Charter that is in Attachment 1 to describe the work problem.

The business case and opportunities were written in detail to avoid doubts among the project team members. Next, SIPOC was developed, a tool that displays the macro steps of the process and its inputs and outputs, as shown in Fig. 2.

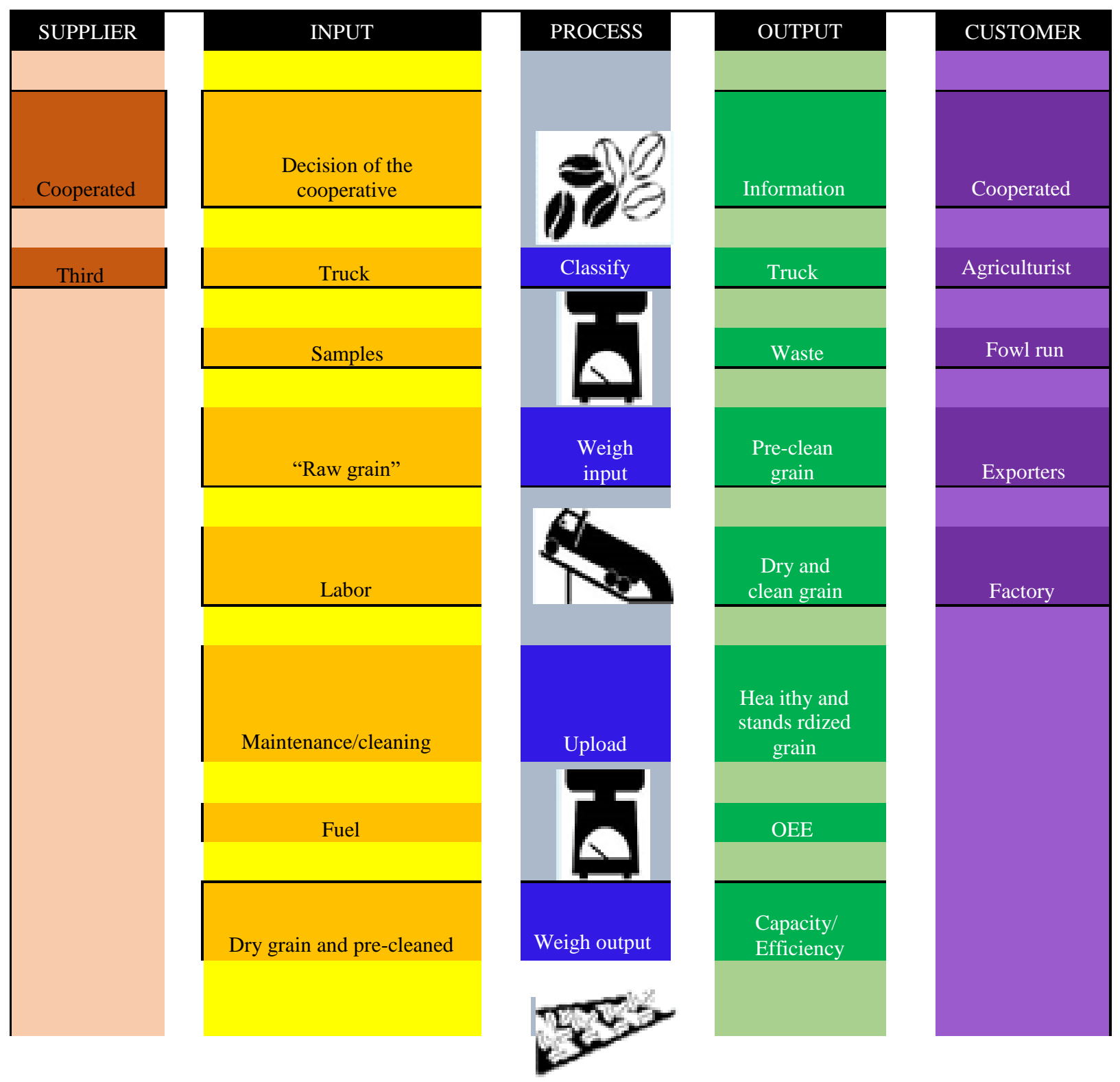

Fig. 2: SIPOC 
The SIPOC analysis should always start with the Process, for subsequent link of suppliers with their process inputs and outputs with end customers. When contextualizing those involved in the process, a macro view is often "masked" within the routines of the employees.

The requirements tree was developed after listening to the Voice of the Customer (VOC) and the Voice of the Business (VOB), enabling to see the restrictions and possible improvements.
As presented by Fig. 3, the changes made by the project cannot affect the safety of the operating units and should not change the standard percentage of product quality, maintaining the cost indicator ( $\mathrm{R} \$ /$ ton); it should improve the grain receipt only. After acquiring some knowledge, it is possible to stratify the $\mathrm{Y}$ of the project, defining which work fronts will be studied and which are not in the scope of the project.

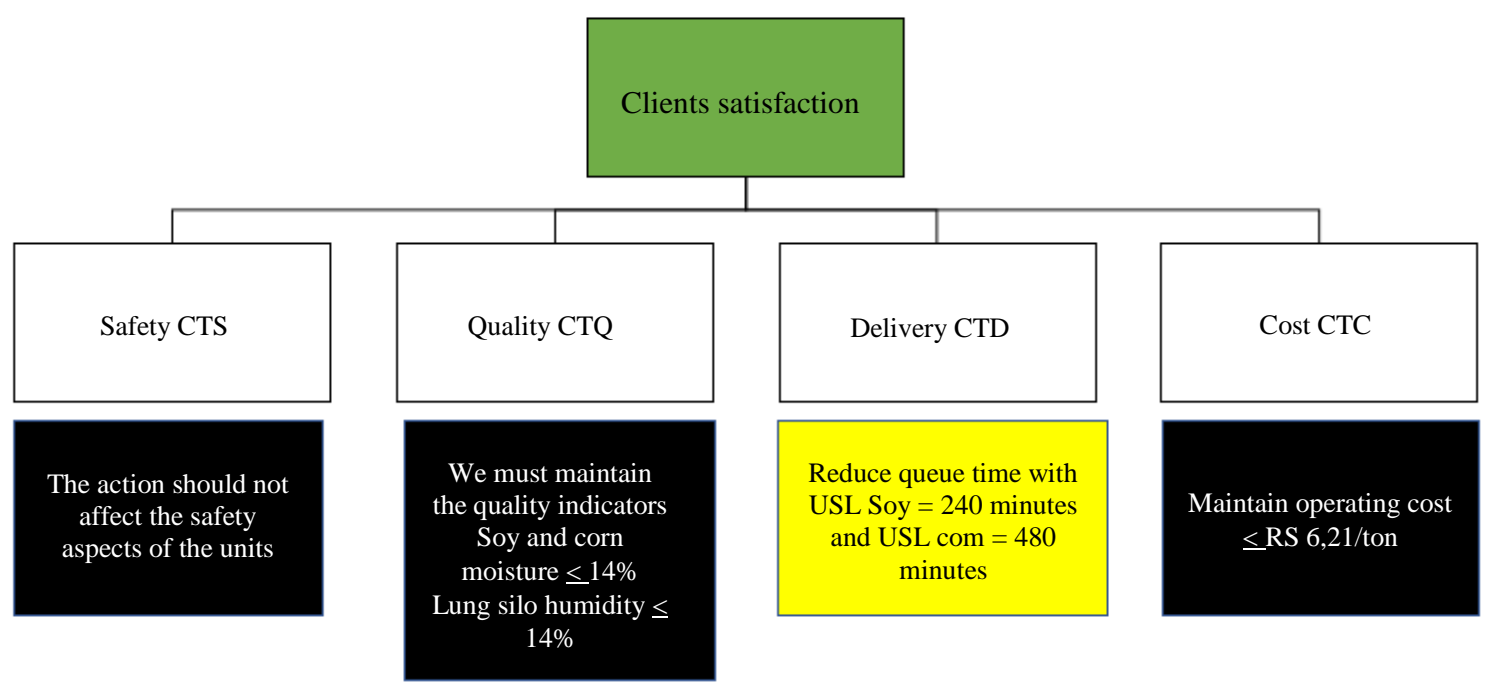

Restrictions

Improve

Fig. 3: Client requirements tree

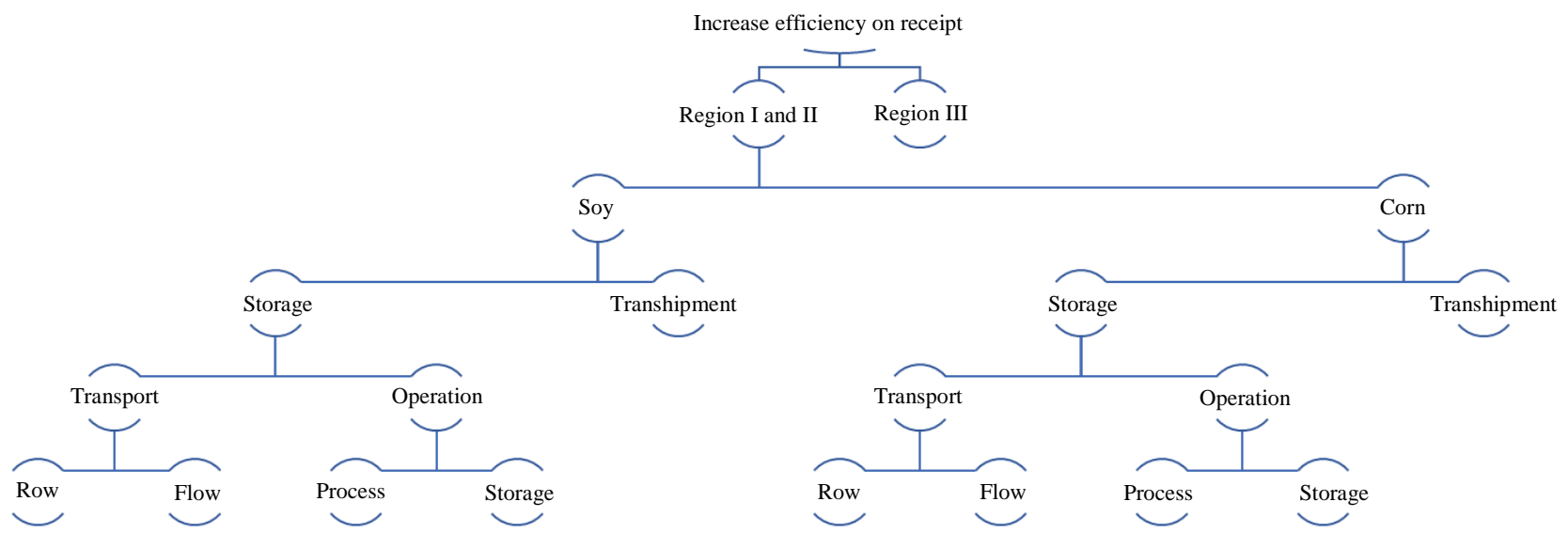

Fig. 4: Y Stratification 
As shown in Fig. 4, Region 3 was excluded from the study, because the products received in this region do not go to the Maringá complex. Due to the distance between them, these products are sold and shipped by the operating units located in Northern Paraná.

The Y stratification resulted in storage units only (Fig. 4), since the products received by them during harvest periods are transported to Maringá between harvests. In these periods, the demand increases and the type of logistic operation has an impact on the expected delivery in Maringá, with increased number of trucks, directly affecting the waiting time in unloading queues. Based on the need to reduce the queue time of the trucks waiting to unload the grains, this continuous improvement project was developed using the Lean Six Sigma methodology, which aims to eliminate adversities and increase process efficiency.

The classification area is the initial stage of the grain receipt process; it classifies and establishes the waste of each cargo according to the results of the moisture and impurity analysis. Fast gain was performed to check for proper classification layout for the sequence of activities performed by the classifiers. To evaluate the efficiency and waste of this process, each classifier was evaluated separately to observe the path traveled by each of them and the total distance in meters. For a better understanding of the process, see following Fig. 5, a sequenced flowchart with its respective steps.

After adding up the distances traveled by the four classifiers to perform a single classification, the total is $111.6 \mathrm{~m}$. Knowing the average cargo to be classified per day, the total daily amount is $13,950 \mathrm{~m}$. The spaghetti diagram of the four classifiers is shown in Fig. 6.

After evaluating the diagrams and the sequence of process activities, together with the daily routine of the employees, the implementation of a new layout for the grain classification area was proposed. This proposal did not demand investments, but adjustments to the physical area of machines and utensils, thus fulfilling the requirements requested by the company. The sum of the distances covered by the four classifiers to perform a single classification after deploying the new layout is $61.4 \mathrm{~m}$. After calculating the total daily amount using the average daily cargo, the result was 7,675 m. Figure 7 shows the spaghetti diagram of the four classifiers with the proposed new layout.

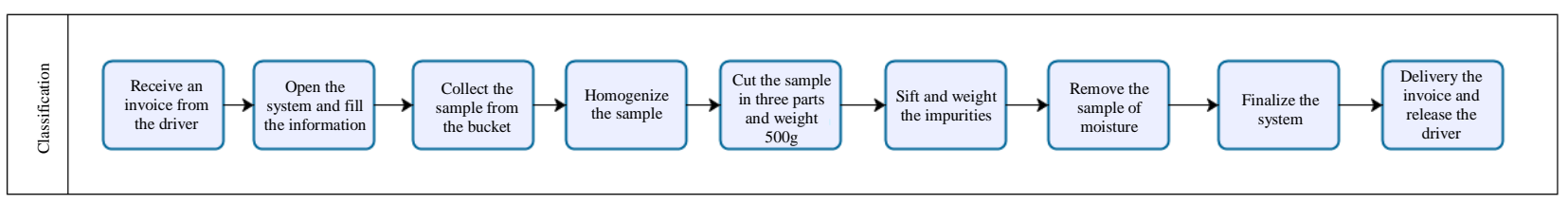

Fig. 5: Process classification

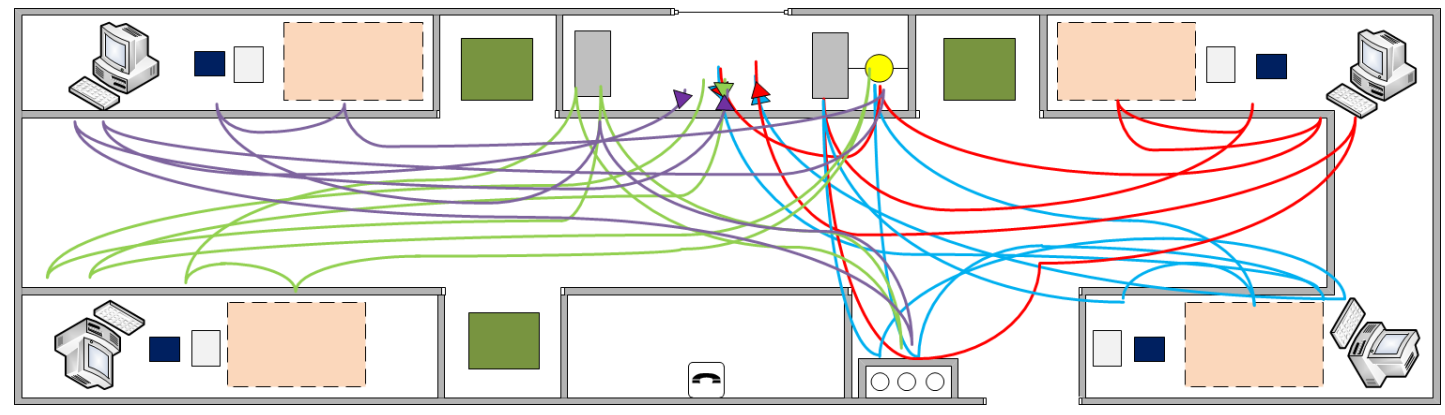

Fig. 6: Spaghetti diagram for the classifiers

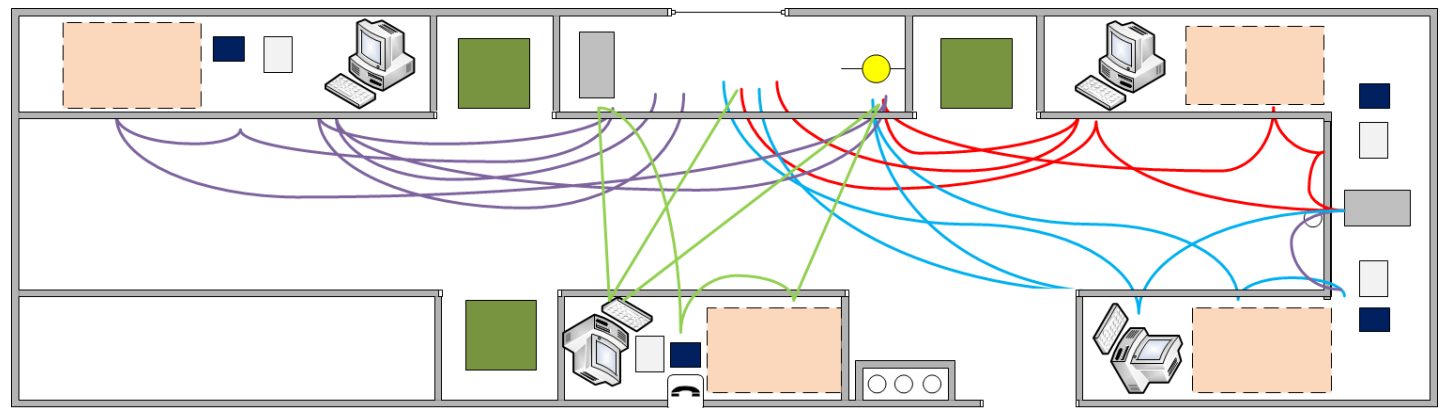

Fig. 7: Spaghetti diagram for the four classifiers - augmented 
The new layout implemented for Grain Classification brought many benefits to the sector - the main one was a $45 \%$ reduction in total handling of the classifiers. Thus, the process became more organized, standardized and efficient; it used to be performed in a random, unadjusted way and with confusing flows that resulted in waiting, queues and a large amount of Work in Process (WIP).

\section{Measure}

The process steps were mapped from the screening yard to the truck exit scale. The time of each step was measured to calculate the efficiency of the grain receipt process. The mapping is in Attachment 2.

The VA time (variables that add value) is the time spent to perform a necessary step of the process, the NVA time is time-demanding activities that do not add values to the customer or business, such as queue, inventory and rework times. Figure 8 illustrates these times. In Table 1 was described the times.

The times were collected through chronoanalyses of 20 samples from each step and the average VA and NVA times were calculated, the VA times do not calculate the displacement times, only the average time of a grain classification and the NVA time calculates the waiting times of trucks between the steps of the production process. The efficiency of the grain receipt process was calculated using the following Equation (1):

$$
\text { Efficiency }=\frac{\text { Time VA }}{\text { Total Time }}
$$

The process efficiency was around $10 \%$, a low percentage for a complex that can store about one million tons. These data indicate that there are many barriers in the process flow, including two intersections at the entrance and exit of the grain classification step between producers associated with the cooperative and the third-party drivers who loaded the products of the operating units of the cooperative.

The entrance of the agricultural producers is through the main gate of the bulk terminal, which is monitored $24 \mathrm{~h}$ a day and is restricted to members and associates of the cooperative. Other drivers who are attracted by higher freight rates in crop seasons enter through the screening yard.

The first crossing in the flow of the cooperative member with the other driver is at the entrance of the classification, as the producers classified their products in the left area and the other drivers in the right area. Besides the contact at the entrance of the classification area, another crossing happened in the flow of the receipt process (illustrated in Attachment 2), at the exit of the classification area, as the cooperative producer, after classifying a sample of its products, is taken to the grain weighing in his exclusive scale located behind the JK warehouse and the third-party drivers are taken the input scale located to the left of the JK warehouse. Although it seems visually simple to identify the crossing found in the flow of grains, day-to-day routines masked the barriers found in the production processes of the cooperative, causing process interruptions.

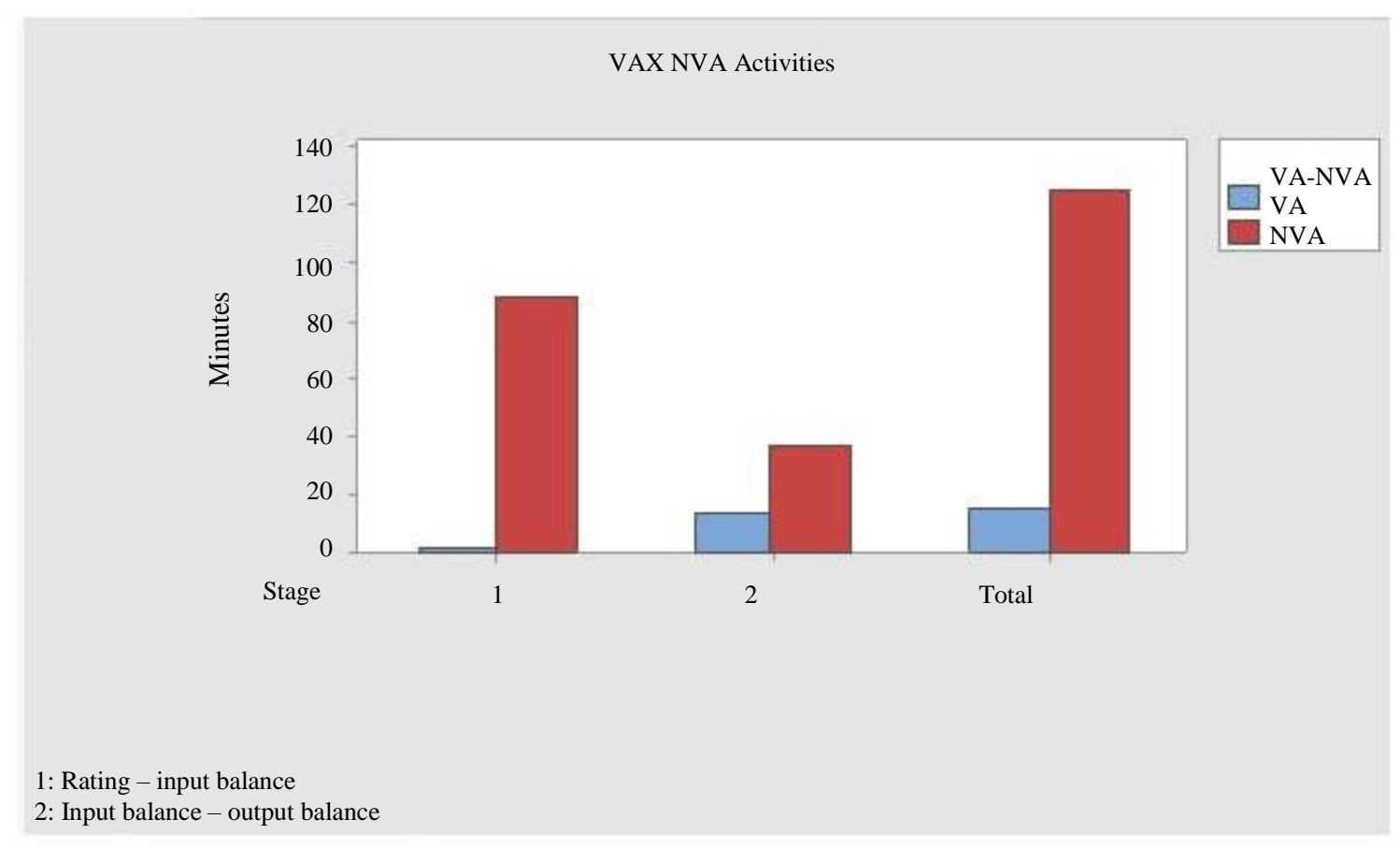

Fig. 8: VA and NVA times of the grain receiving process 
Even after demonstrating the crossings in the flows, there was resistance on the part of the classifiers and people in charge of the bulk terminal, as they said a Btrain truck coming from the screening yard would not be able to perform a curve maneuver. Thus, tests were conducted to check if in fact larger trucks would not be able to perform the maneuver. The B-train truck test was successfully performed and as the truck was able to easily enter the site previously used by the producers, the test was validated and presented to those in charge of the bulk terminal. Then, the flows were exchanged, ending the crossing that existed in the grain classification area, as illustrated in Fig. 9.

\section{Analyse}

At this stage, the project team should find solutions, using creativity and exchanging experience among the members.

First, wood fence posts were installed to divide into three lanes the way leading to truck entrance. To control the flow in a 'pulled' manner and no longer in a 'pushed' one, three gates were installed to release one truck at a time to be classified. After the classification, about 400 wood fence posts were installed to organize the queues, with signs displaying the names of the complexes where the trucks should unload the products after weighing on the scale.
After weighing, the trucks unloaded the products in the JK and LM complexes in their respective hoppers, $12,13,14$ and 9,10 . Then, they were directed to the exit scale where they weighed the trucks to confirm truck unloading of grains. To automate part of this process, the system was adapted at the gates so when a truck left the exit scale, the gate of the entrance scale was released, pulling the next truck in the queue. At this step, it was important to consider all possible risks, so the FMEA was performed. Based on the three steps of the process: Screening, classification and unload, 11 macro activities were defined and 30 failure modes were prioritized. Then, the 5 Whys were conducted to find possible solutions. Using the 5 Whys methodology, 98 whys were performed and 31 solution actions were generated. The root causes were identified as shown in Table 2 .

The need to reduce the queue time to increase grain receipt efficiency was clear, so it was necessary to eliminate conflicts and pull-down barriers that affected the flow of the studied process. Besides the changes previously made, a proposal suggested to limit the number of trucks inside the complex, called WIP (work in process), that is, the number of trucks from the classification to the exit scale. Another solution was the standardization of all activities of the hoppers, who is the employee responsible for operating the truck tilter.

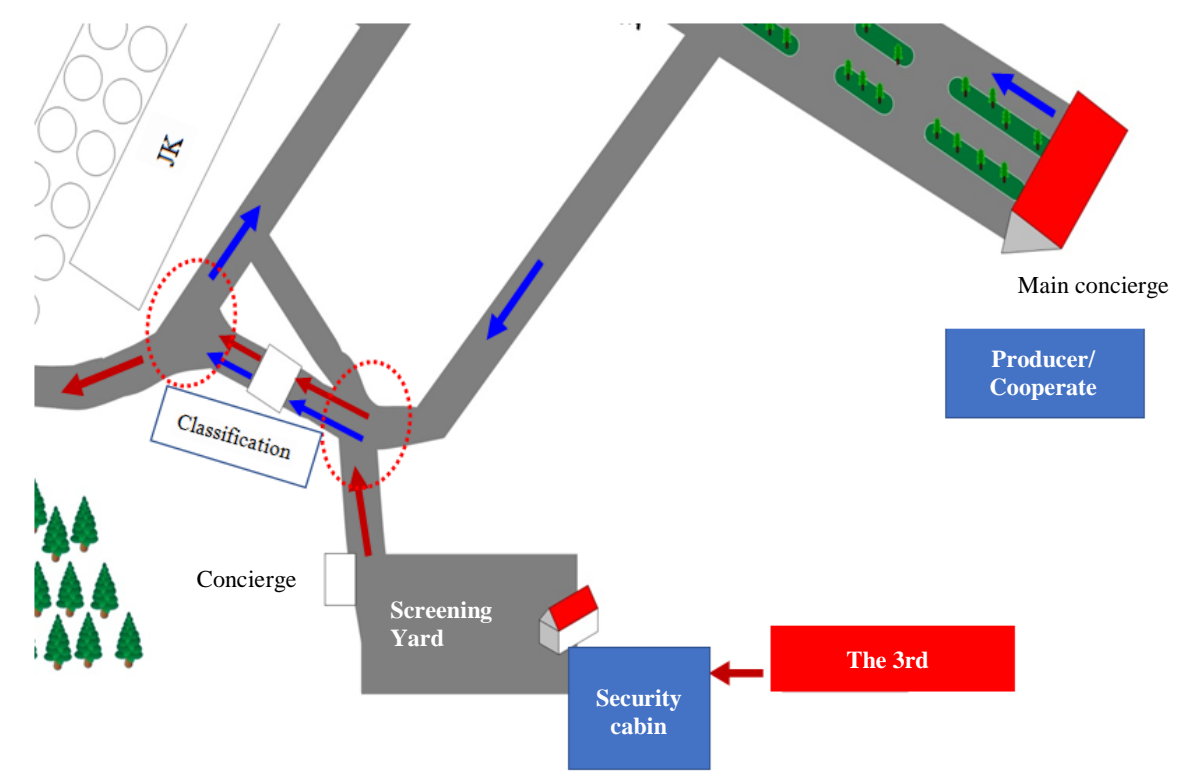

Fig. 9: Direction of flows among the participants of the grain classification stage

Table 2: Root causes analysis

\begin{tabular}{lllll}
\hline Number & "Y" Variable & "X" Variable & Analysis & Detected Effect \\
\hline 1 & Queue Time & Trucks & Wait in Line & Inefficiency \\
2 & Work in Process (WIP) & Minutes & Trucks Inventory within the process & Inefficiency \\
3 & Tons & Days & Daily tons allowance & Bottlenecks \\
\hline
\end{tabular}


The Analyze step compiled data and generated action plans to support the implementation of improvements in the following phase.

\section{Improve}

In this step, the project team started to implement the suggested improvements identified in the previous phase.

Raised platforms were installed, so third-party trucks were isolated in 3 organized lines. Then, associated producers would have exclusivity in the right-side line, because there would be no more crossing, ending conflicts between the drivers.

When the truck leaves the classification area, it takes the invoice showing cargo classification and unload site, that is, the hopper number it should use. This ticket shows the queue the truck should follow to reach its destination. After the truck goes to the correct queue, it will be obliged to follow the isolated route through the steps until it arrives at the traffic light installed at the entrance of the scale. Three traffic lights with 3 gates were installed so that the scale operator can control the weighing flow.

The gates were interconnected with the current exit scale gates, so after cargo unloading and truck weighed at the exit scale, the gate of the queue in which the truck was before weighing can release the next truck. After weighing the truck at the entry scale, the driver unloads the product in the hopper indicated on the ticket. As analyzed in the previous step, the hopper operators did not have a work instruction, so these instructions were developed in a video showing the sequence of activities.

\section{Control}

In this step, it is important for the project team to involve the process owner in the improvements, as the team starts to leave the project area and the improvements cannot be lost over time. Then, the bulk terminal manager who is the owner of the grain receipt process was involved to develop a control matrix, which is the tool that concentrates all controls, with those in charge of ensuring the improvements.

The controls were divided into areas such as: Maintenance, Classification, Scale and Management and designed as a flow so that the processes have greater synergy and alignment during the control actions. This matrix focuses on the main indicators, those responsible for monitoring, the period and a contingency plan, that is, a plan to prevent common causes and control actions, if necessary. After the improvements implemented with the project, there was a reduction in the waiting time of the trucks to unload the products, as presented in Fig. 10.

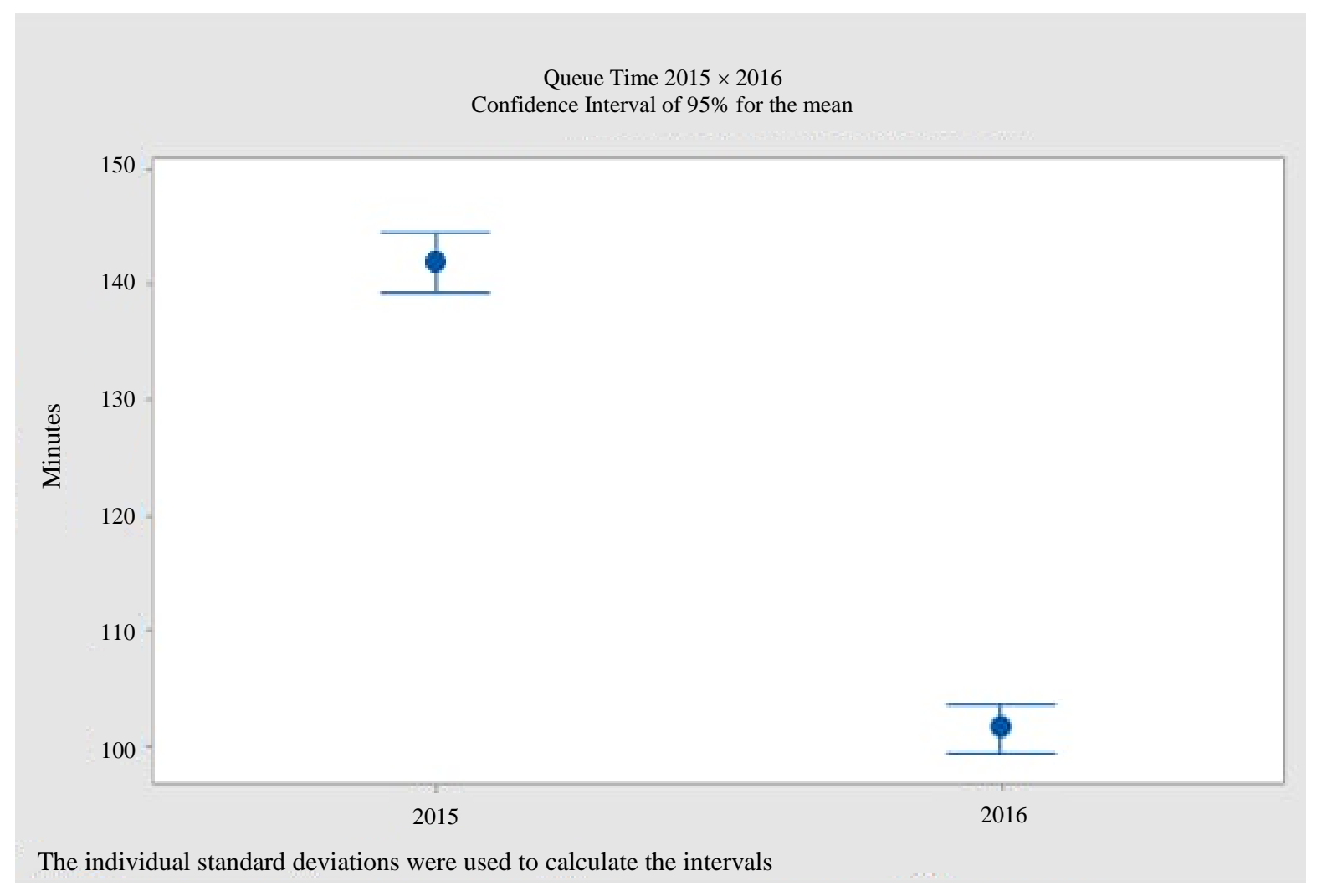

Fig, 10: Queue time reduction $(2015 \times 2016)$ 
The reduction was around 40 min per truck, about $28 \%$ of the waiting time, that is, for every three unloading trucks, it is possible to receive another one. This time will improve when maintenance and drying efficiency projects are concluded, as they are solving the downtime due to equipment breakdown and increasing the drying capacity of grains - the latter affects product unloading, because when the dryer is full, the hopper chute no longer feeds product and soon the hopper is full, preventing the next truck unloading. The number of trucks inside the bulk terminal is being controlled, with an upper limit of 60 trucks, as shown in Fig. 11.

Figure 11 shows data related to the 2015 and 2016 soybean crops. Each marked point refers to one truck at a $5 \mathrm{~min}$ frequency, that is, every $5 \mathrm{~min}$ the system shows us how many trucks are in the facilities from the grain classification area until product unloading, being the limit 60 trucks inside the bulk terminal. However, the best improvement was the bulk terminal organization and signs, because the crossing flows caused many conflicts that exhausted the employees. And now with tracked number of trucks inside the bulk terminal, problems like truck breakdown are being identified and resolved faster. To verify the efficiency of the changes, a satisfaction survey was conducted with the drivers to evaluate the improvements implemented, illustrated in Fig. 12.

As conducted before, a chronoanalysis was performed to verify the reduction of time, from the classification step to the exit scale. The displacement from the classification area to the entrance scale had a $77 \%$ reduction in relation to Table 3 , which shows the times before the improvements. This reduction was due to a new possibility to segregate the products that are transported by the trucks transport in queues to the unloading place.

Table 3: VA and NVA time of steps after the improvements

\begin{tabular}{lcc}
\hline Stage & VA Time (min) & NVA Time (min) \\
\hline Classification - Input Balance & 2 & 57 \\
Input Balance - Output balance & 13 & 27 \\
Total & 15 & 84 \\
\hline
\end{tabular}

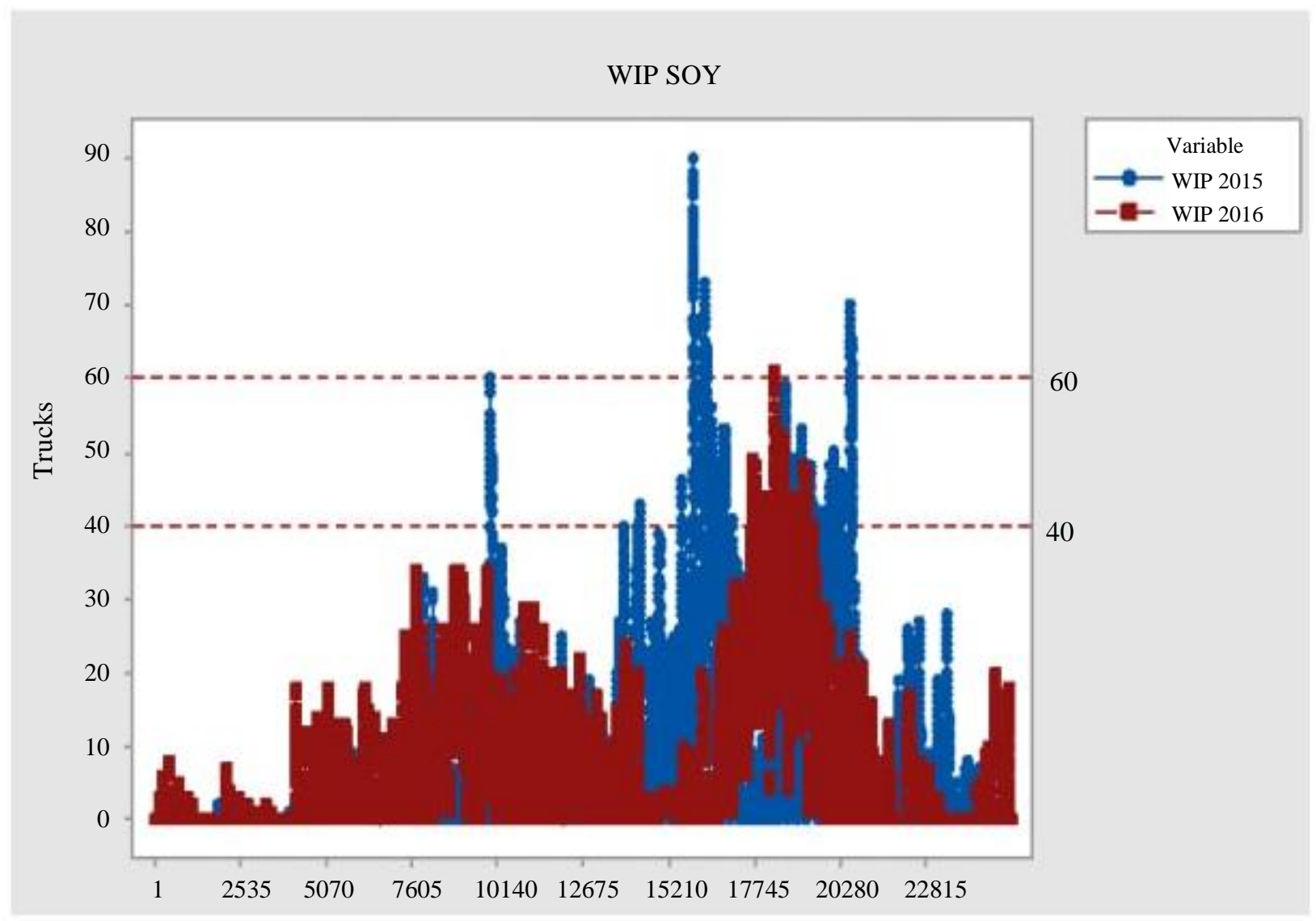

Fig. 11: Number of trucks inside of bulk carrier 


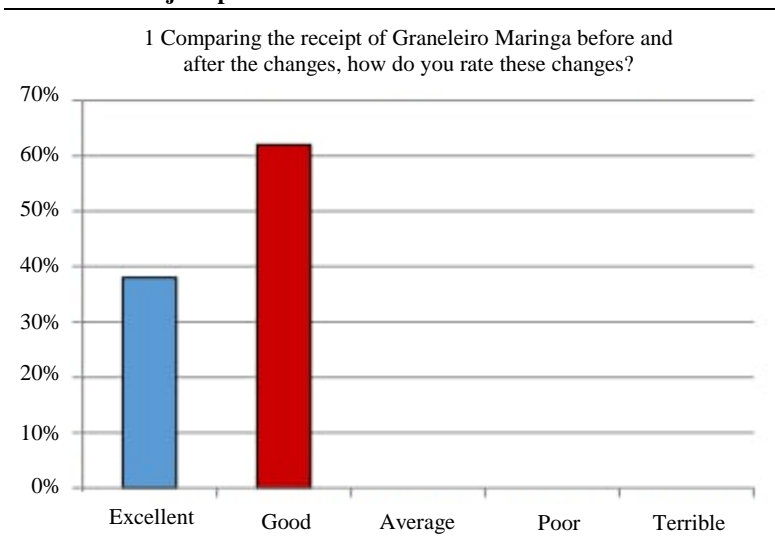

2 Which benefit was more representative?

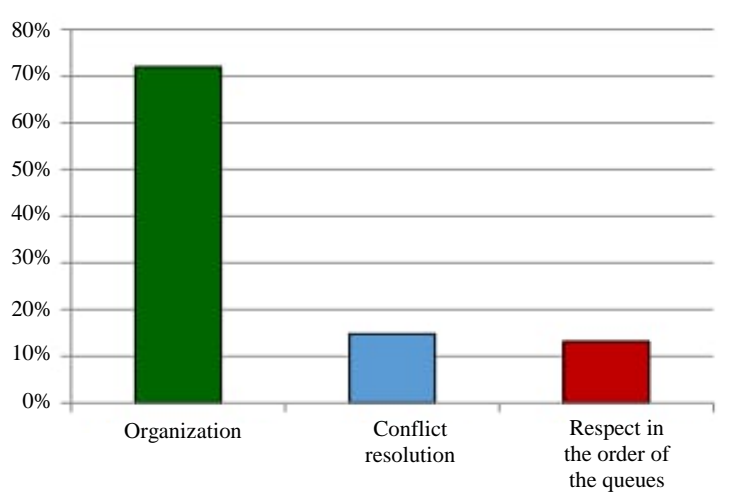

Fig. 12: Satisfaction survey data

\section{Conclusion}

Due to the high competitiveness for an increased market share, companies tend to invest in cost reduction projects, eliminating waste and increasing customer satisfaction.

Based on this concept, this study shows that improvement projects are effective, with data and facts that Lean Six Sigma can be implemented in any business area, not only in industries, breaking cultural paradigms related to the application of this tool. The project managed to apply LSS to a bulk terminal of an agroindustrial cooperative and brought several benefits, increasing the satisfaction of drivers and employees involved in the grain receipt process.

At first, resistance was felt from former employees in the cooperative, as they were in the comfort zone, showing no interest in changing. However, with time, employees changed their view and now they feel grateful for the improvements implemented, since employees who did not believe in improvements now recognize that dayto-day work has become easier and faster, not wasting time with activities that do not add values to customers.

The raised platforms implemented in the bulk terminal allow product segregation by type in different queues, separating them as "lanes" and optimizing the grain drying process, due to the separation of wetter products from less wet ones in different queues; thus each grain dryer dries a more uniform amount of product of similar moisture. This study enabled to consolidate the idea that Lean Six Sigma projects can be part of improvement studies in bulk terminals of agroindustrial cooperatives, starting a new wave of related projects such as: Cost management and active equipment management.

\section{Author's Contributions}

Seiti Moraes Sakumoto: Development work and conducted analysis of the research.

Ana Caroline Dzulinski: Data analysis.
Daiane Maria De Genaro Chiroli: Provided the research topic and guided the research development, experimental plan and data analysis. All authors contributed to the writing of the manuscript.

\section{Ethics}

This article is an original research paper. There are no ethical issues that may arise after the publication of this manuscript.

\section{References}

Adams, C., P. Gupta and C. Wilson, 2003. Six Sigma Deployment. 1st Edn., Butterworth-Heinemann, Oxford, ISBN-10: 0750675233, pp: 290.

Alhuraish, I., C. Robledo and A.A. Kobi, 2017. Comparative exploration of lean manufacturing and six sigma in terms of their critical success factors. J. Cleaner Product., 164: 325-337. DOI: 10.1016/j.jclepro.2017.06.146

Antony, J., 2005a. Assessing the status of six sigma in the UK service organizations. Proceedings of the 2nd National Conference on Six Sigma, (CSS' 05), Wroclaw, pp: 1-12.

Antony, J., 2005b. Six Sigma for service processes. Bus. Process Manage. J., 12: 234-248. DOI: $10.1108 / 14637150610657558$

Antony, J., E.V. Gijo, V. Kumar and A. Ghadge, 2016. A multiple case study analysis of six sigma practices in Indian manufacturing companies. Int. J. Quality Reliability Manage., 33: 1138-1149. DOI: 10.1108/IJQRM-10-2014-0157

Antony, J., M. Kumar and C.N. Madu, 2005a. Six Sigma in small and medium sized UK manufacturing enterprises: Some empirical observations. Int. J. Quality Reliability Manage., 22: 860-874.

DOI: $10.1108 / 02656710510617265$ 
Antony, J., M. Kumar and M.K. Tiwari, 2005b. An application of Six Sigma methodology to reduce the engine overheating problem in an automotive company. IMechE - Part B, 219: 633-646.

DOI: $10.1243 / 095440505 X 32418$

Azadeh, B. Nasirian, V. Salehi and H. Kouzehchi, 2016. Integration of PCA and DEA for identifying and improving the impact of Six Sigma implementation on job characteristics in an automotive industry. Quality Eng., 29: 273-290.

DOI: 10.1080/08982112.2016.1182633

Barros, G.S.C., 2006. Brazilian Agribusiness: Perspectives, challenges and diary for its development. ESALQ/USP, CEPEA, Piracicaba.

Biazetto, F. and D.M.G. Chiroli, 2015. Application of the Six Sigma methodology to reduce the loss of soybean transfer in a grain processing unit. (Course Completion Work) Department of Production Engineering. State University of Maringá, Maringá - Paraná.

Carvalho, M.M., 2002. Selected Six Sigma Projects. In: Six Sigma Management Strategy for Process Improvement, Rotondaro, R. G. (Org.), Products and Services. Atlas, São Paulo, pp. 49-79.

Connor, G., 2003. Benefiting from Six Sigma. Manufact. Eng., 130: 53-59.

Domenech, C., 2016. Building Green Belts: The Lean Six Sigma Strategy for Continuous Improvement. M.I. Consultants Domenech.

Gijo, E.V., R. Palod and J. Antony, 2018. Lean Six Sigma approach in an Indian auto ancillary conglomerate: A case study. Product. Plann. Control, 29: 761-772. DOI: 10.1080/09537287.2018.1469801

Dahlgaard, J.J. and S.M. Dahlgaard-Park, 2006. Lean production, six sigma quality, TQM and company culture. TQM Magazine, 18: 263-281. DOI: $10.1108 / 09544780610659998$

Fursule, N.V., S.V. Bansol and S.N. Fursule, 2012. Understanding the benefits and limitations of six sigma methodology. Int. J. Scientific Res. Public., 2: $1-9$.

Gupta, N. and P.K. Bharti, 2013. Implementation of six sigma for minimizing the defects rate at a yarn manufacturing company. Int. J. Eng. Res. Applic., 3: 1000-1011.

Harry, M. and R. Schroeder, 2000. Six Sigma: The Breakthrough Management Strategy Revolutionizing the World's top Corporations. 1st Edn., Currency, New York, ISBN-10: 0385494378, pp: 300.

Harry, M.J., 1998. Six Sigma: A breakthrough strategy for profitability. Quality Progress, 31: 60-65.

Hilton, R., M. Balla and A.S. Sohal, 2008. Factors critical to the success of a Six-Sigma quality program in an Australian hospital. Total Quality Manage. Bus. Excellence, 19: 887-902.

DOI: $10.1080 / 14783360802224396$
Kuvvetli, U. and A.R. Firuzan, 2017. Applying Six Sigma in urban public transportation to reduce traffic accidents involving municipality buses. Total Quality Manage. Bus. Excellence, 30: 82-107. DOI: 10.1080/14783363.2017.1297198

Kwak, Y. and F. Anbari, 2006. Benefits, obstacles and future of six sigma approach. Technovation, 26: 708-715.

Laureani, A. and J. Antony, 2016. Leadership - a critical success factor for the effective implementation of Lean Six Sigma. Total Quality Manage. Bus. Excellence, 29: 502-523. DOI: 10.1080/14783363.2016.1211480

Mamatha, K. and H.V. Vasuki, 2014. Application of six sigma methodology to reduce rework at earth moving equipments. Int. J. Eng. Sci. Technol., 5: 112-120.

Tanik, M. and A. Sen, 2012. A six sigma case study in a large-scale automotive supplier company in Turkey. Total Quality Manage. Bus. Excellence, 23: 343-358. DOI: 10.1080/14783363.2011.637798

Nakhai, B. and J.S. Neves, 2009. The challenges of Six Sigma in improving service quality. Int. J. Quality Reliability Manage., 26: 663-684. DOI: 10.1108/02656710910975741

Pande, P.S., R.P. Neuman and R.R. Cavanagh, 2000. Six Sigma Strategy. Like GE, Motorola and other large companies are sharpening their performance. Qualitymark, Rio de Janeiro, pp. 472.

Pamfilie, R., A. Petcu and M. Draghici, 2012. The importance of leadership in driving a strategic Lean Six Sigma management. Proc. Soc. Behav. Sci., 58: 187-196. DOI: 10.1016/j.sbspro.2012.09.992

Prashar, A., 2016. Six Sigma adoption in public utilities: A case study. Total Quality Manage. Bus. Excellence, 27: 479-506.

DOI: $10.1080 / 14783363.2015 .1014782$

Pisani, M.J., R. Hayes, A. Kumar and L. Lepisto, 2009. Is Six Sigma culture bound? A conceptual model and propositions for further inquiry. Total Quality Manage., 20: 1123-1137.

DOI: $10.1080 / 14783360903246884$

Roberts, C.M., 2004. Six Sigma signals. Credit Union Magazine.

Sastry, M.N.P., M.D. Devi and E.S. Reddy, 2011. Application of six sigma for process improvement and variation reduction of automotive batteries. Sci. Insights: Int. J., 1: 25-31.

Snee, R.D., 2004. Six Sigma: The evolution of 100 years of business improvement methodology. Int. J. Six Sigma Competitive Advantage, 1: 4-20. DOI: 10.1504/IJSSCA.2004.005274 
Snee, R.D., 2010. Lean Six Sigma-getting better all the time. Int. J. Lean Six Sigma, 1: 9-29. DOI: $10.1108 / 20401461011033130$

Tenera, A. and L.C.A. Pinto, 2014. Lean Six Sigma (LSS) project management improvement model. Proc. Soc. Behav. Sci., 119: 912-920. DOI: $10.1016 /$ j.sbspro.2014.03.102

Uluskan, M. and N. Erginel, 2017. Six Sigma experience as a stochastic process. J. Quality Eng., 29: 291-310.
Voelkel, J.G., 2017. Meeting overall reliability requirements for heats of steel: A case study. Quality Eng., 29: 446-454.

DOI: $10.1080 / 08982112.2017 .1317803$

Watson, J.J., 2003. Satisfaction through Six Sigma. Eng. Syst., 20: 94-98.

Zhang, Q., M. Irfan, M. Khattak, X. Zhu and M. Hassan, 2012. Lean Six Sigma: A literature review. Interdisciplinary J. Contemporary Res. Bus., 3: 599-605. 